\title{
Beyond why to what and how: the use of systems thinking to support problem formulation in systems engineering applications
}

\author{
S. Elsawah, A. McLucas and M. Ryan \\ School of Engineering and Information Technology, University of New South Wales, Canberra \\ Email: s.elsawah@unsw.edu.au
}

\begin{abstract}
Systems thinking is a transdisciplinary research field which provides a powerful framework (i.e. theories, methodologies, and methods) for systems engineers to deal with the complexities of problem formulation. In this paper, we are motivated by the valuable benefits that systems thinking can contribute to theory and practice of requirement engineering. At a theoretical level, systems thinking is an intellectually rich domain of theoretical stances on how to build a systemic understanding of complex situations. Each of these theories offers a unique perspective on the problematic situation. For practice, using a systems approach offers a variety of approaches and techniques to serve different purposes (e.g. exploring purpose, bringing in different viewpoints, building consensus) and problem framing phases (e.g. identify issues, assess alternative solutions). This paper is intended for system engineers (researchers, practitioners, students) who are interested to know about systems thinking, and how it may contribute to understanding and framing complex situations, and identifying stakeholders' needs. First, we review the literature on systems thinking in the systems engineering field. We provide a discussion of these studies as there have been limited discussions and understanding of the use of systems thinking in the systems engineering literature. The overview of literature shows a gap in applications of the systems thinking ideas and methods in literature. Second, we develop a preliminary framework which brings together a holistic repertoire of systems thinking concepts and methods from different paradigms. The primary purpose of the framework is to: (1) provide a systems thinking-grounded view of the requirement elicitation process, (2) raise awareness about the variety of available systems thinking, their theoretical background, and how they can contribute to the process, and (3) provide insight into the practice aspects of these methods, including their: strengths, limitations, expected outcomes, and potential of combining them with other methods. The framework is based on critical systems practice theory. In practice, whenever faced with a task in a particular situation through inquiry process, a practitioner who is aware of the various theories and is equipped with an armory of methods and tools, will be able to assess the fitness of methods to the context. As the situation changes, some issues disappear and new issues emerge, the analyst will continually cycle around the phases of creativity, choice, and implementation in an ongoing quest for learning about the situation and the purpose of the proposed solution. The presented framework is theory-driven, and has not been applied and tested in real-life systems engineering applications. Future applications will help improve the framework based on an empirical understanding of 'what works' and 'what does not work' in 'what context'.
\end{abstract}

Keywords: Systems thinking, systems engineering, critical systems thinking, requirements elicitation, needs analysis 


\section{INTRODUCTION}

In the early problem solving literature, systems engineering has been pigeon-holed as a problem solving methodology appropriate for situations described as: simple (i.e. stable and predictable) and unitary (i.e. decision makers agree on goals and solutions). This view may be outdated and does not reflect the increasing complexity of "contemporary systems engineering" endeavors (Cook and Ferris, 2007), especially with the emergence of the 'system of systems' systems engineering (Katina et al., 2014). It is widely acknowledged that systems engineering projects operate in increasingly complex environment where systems engineers are required to address vexing socio-technical challenges that extend far beyond effectively conducting the technical aspects of the design-build-test process (Baxter and Sommerville, 2011). To name a few of these challenges:

- The complex and interactive factors (technical, social, organizational, and personal) that influence the solution success, and those many of which cannot be predicted and accounted for in the design and implementation;

- The increasing pressure to delivers according to a diverse, sometimes conflicting, range of views and expectations surrounding the solution's goals;

- Increasing size and multi-disciplinary nature of teams and stakeholders involved a system engineering project

In response to recognizing these challenges, three key related ideas relevant to this article have emerged in the systems engineering field. First, the complexity of situations that systems engineers encounter has highlighted the criticality of understanding 'the right problem right' as a basis for developing effective solutions. Second, traditional requirement engineering methods may not be entirely sufficient to provide the required understanding of complex social and organizational issues. Finally, there is a need to explore new epistemologies and methodologies as means to help bridge the gap (Hitchins, 2015).

Systems thinking is a transdisciplinary research field that can contribute to meeting the need for improved problem framing and understanding as a basis for successful requirement engineering. Systems thinking is uniquely positioned to address the challenges of problem framing as its central focus is on understanding complex problems which beg for systemic solutions. Systems thinking provides a powerful framework (i.e. theories, methodologies, and methods) for systems engineers to deal with the complexities of problem formulation. This has the potential to deliver improved project outcomes (e.g. meeting clients' expectations).

In this paper, we are motivated by the valuable benefits that systems thinking can contribute to theory and practice of requirement engineering. At a theoretical level, systems thinking is an intellectually rich domain of theoretical stances on how to build a systemic understanding of complex situations. Each of these theories offers a unique perspective on the problematic situation. For practice, using a systems approach offers a variety of approaches and techniques to serve different purposes (e.g. exploring purpose, bringing in different viewpoints, building consensus) and problem framing phases (e.g. identify issues, assess alternative solutions).

\section{SYSTEMS THINKING IN SYSTEMS ENGINNEERING}

The first shaking call for the need of systems thinking is the pioneering work of Peter Checkland. Being a systems engineer himself, Checkland, (2000, p.S1) wrote "We found that although we were armed with the methodology of systems engineering and were eager to use its techniques to help engineer real-world systems to achieve their objectives, the management situations we worked in were always too complex for straightforward application of the systems engineering approach".

Although not directly interested in systems thinking per se, Bickerton and Siddiqi (1993) developed a preliminary classification of requirement engineering methods to include few systems methods. They broadly categorized methods into hard, soft, critical, dual, network, democratic, and post-modern. However, some of the methods classification is not consistent with how they are classified in the systems thinking literature. For example, they classified cognitive mapping as a hard method. Hitchin (2007) heavily advocates the use of soft systems methods a "frontend" stage for the systems engineering process. He presented system dynamics and SSM as two useful methods. He also developed the Rigorous System Modelling (RSM) as a process for formulating problems in systems engineering projects. Kasser and Makley (2008) defined a set of nine “system thinking perspectives' (STP) to guide system engineers to think about a particular problem from different angles, including: operational, functional, big picture, structural, generic, continuum, temporal, 
quantitative, and scientific perspectives. Katina et al. (2014) explored the challenges related to the requirement elicitation in complex situation, including: the role of the observer, the nature of the system requirements, and the influence of the system environment. They discussed the limitations of traditional requirement elicitation approach to deal such challenges, and suggest the need for an approach grounded in systems theory to support requirement engineering. They presented a framework of three steps to support robust requirement elicitation process. These steps are: understanding the nature of the system observer, understand the nature of requirements, and understand the nature of the environment of the requirements. They indicate that the framework is still highly conceptual, and need to be elaborated with appropriate methods and tools.

Against the backdrop of this literature, we have the following observations on the contribution of systems thinking to systems engineering. First, it is scattered and one-off attempts without a clear research agenda. Second, the argument for using systems thinking is largely conceptual and more focused on justifying the rationale for a system thinking approach rather with little guidance on what methods are available and how they can be used. Applications of systems thinking methods (mostly SSM) are still very limited. We strongly agree with Hitchin (2015) who argues that the use of systems methods in systems engineering barely scratches the surface, and there is a need to explore the use of new methods and, as we would like to add, new epistemologies to help improve the theory and practice of problem formulation. This motivation sparks the development of the framework to be presented in the following section.

\section{PROPOSED FRAMEWORK}

\subsection{Overview}

In this section, we present an overview of the proposed framework (See Figure 1). Here, we are concerned with complex situations where the systems engineer wishes to use a systems approach to understand the problem on hand and define stakeholder's needs. We frame the frontend problem definition process as a multi-methodology inquiry process where the purpose is to explore and understand the problem situation and potential solutions. In a multi-methodology process, the analyst selects and combines methods from different methodologies and paradigms in order to help understand the situation from multiple perspectives and gain a more comprehensive appreciation of the situation. For example, the case when the analyst is faced with a situation where stakeholders have different and, may be conflicting, views regarding the problem (e.g. the design of supervised drug injection facility). To define the problem, the analyst may need to use a soft systems method to elicit and analyze subjective perspectives of individuals (e.g. drug users) and interest groups (e.g. doctors). Also, he/she may still need to use a critical systems approach to ensure that the project boundaries are sufficiently inclusive and no values or interests have been marginalized which may endanger the long term sustainability of the proposed solution. However, this is not sufficient, as he/she still needs to use a hard systems model (e.g. simulation model) to assess the performance of design alternatives. The proposed framework brings together methods from different paradigms, and therefore, widens the variety of methods to be used in the process of inquiry.

Three critical systems thinking theories have guided the development of the framework: Bower's multiparadigm framework, Jackson's critical systems practice, and Minger's multi-method. First, in his attempt to bring different together in a theoretically coherent and pragmatic way, Bower argues there are two types of process and structures (not systems) surrounding us: type 1 are 'real' and exist for their own right independent from an observer-specific attributes (e.g. views). Type 2 structures and processes are dependent on an observer-specific attributes. Bower agrees with Jackson that a paradigm is separate from the real world. When we adopt a particular paradigm, we define and use systems concepts according to this paradigm in order to examine the problem situation. For example, we use the soft systems methodology to define the system as a learning inquiry. We used Bower's ideas to define the ontological, epistemological, and axiological assumptions as in the paradigm part of Figure 1. The assumptions underpinning the framework do not oblige the analyst to commit to any particular paradigm viewpoint. The analyst has the flexibility to adopt a particular epistemology and ontology as long as he/she is aware of the underpinning theoretical 
heritage and implications to practice. Second, we used Jackson's (2006) critical system practice to organize the inquiry process into four phases: creativity, choice, implementation, and reflection (as shown in the metamethodology part of Figure 2). Third, we used the methodology decomposition map (the whole layout of Figure 1) from Minger's multi-methodology theory to represent the framework. The methodology decomposition map is a holistic way of showing the relationship between the philosophy (i.e. why), the methodology (i.e. what), and methods/tools (i.e. how).

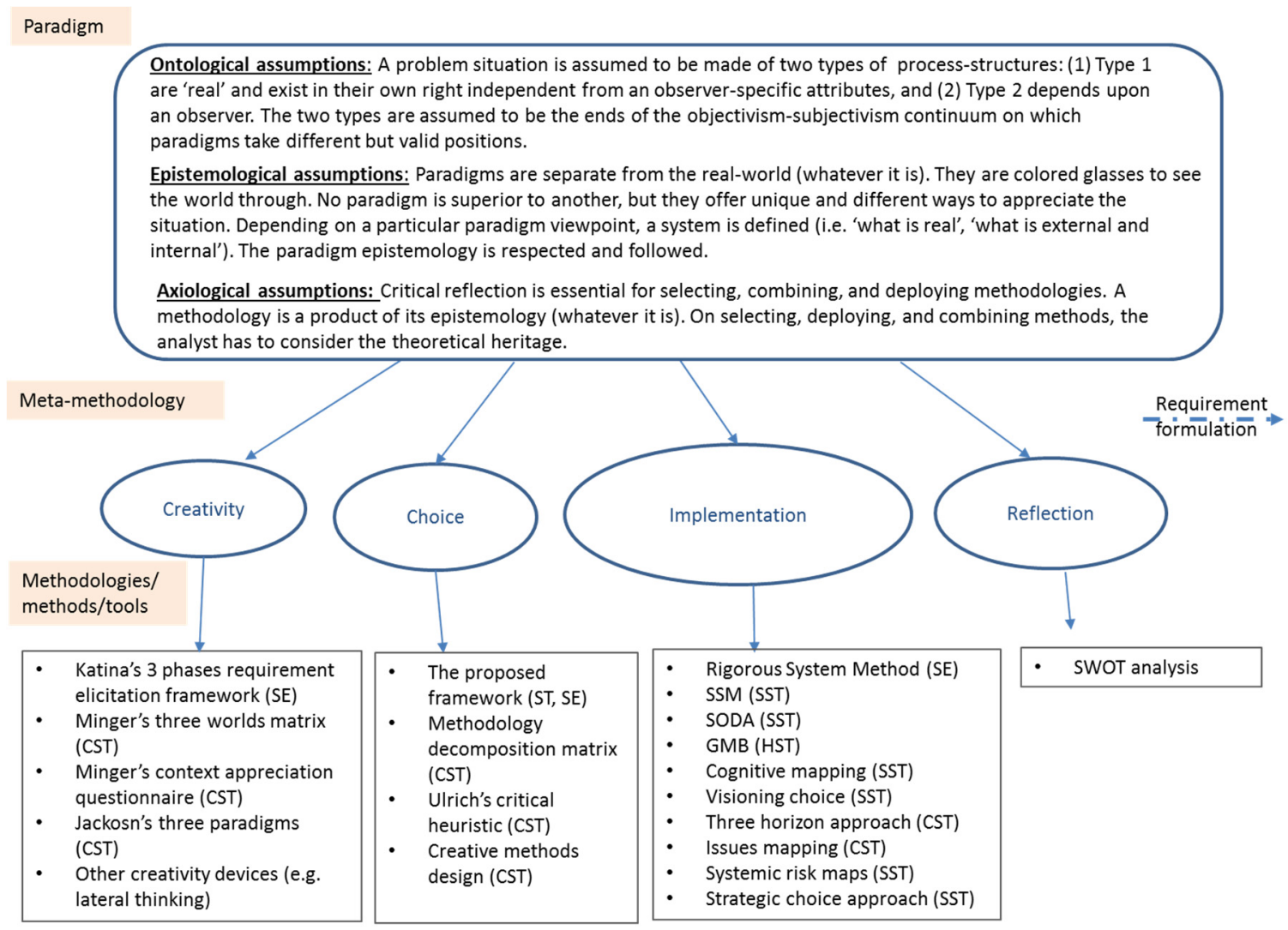

Figure 1: The proposed framework (work in process)

\subsection{Creativity phase}

The role of creativity in problem solving goes back to Ackoff's (1979) seminal book on the Art of Problem Solving. There are different definitions of creativity, but we find the most relevant to the article is the one presented by Hermann (1996) who described creativity as: 'Among other things, it is the ability to challenge assumptions, recognize patterns, see in new ways, make connections, take risks, and seize upon chance'. This embodies the notion of being open to look from different perspectives and use new methodologies. In order to appreciate the complexity and diversity of the problematic situation, the analyst needs to think creatively about the problem in the sense of getting exposed to different viewpoints before committing to a particular stance. The analyst is encouraged to use various creativity devices to make sense of the situation and explore the need and potential value of employing a systems thinking approach.

In Creative Systems Practice method, Jackson (2006) suggests that the use of the four paradigms (functionalist, interpretive, emancipatory and postmodern) must be considered to ensure that different epistemological positions have received sufficient attention. In doing so, the analyst should not consider these paradigms in the sense of 'either...or' road fork. Instead, he/she needs to think of these paradigms as different lenses or glasses for viewing the problematic situation which offer a unique perspective to the inquiry process. To inform practice, he/she needs to think of the learning benefits from wearing a paradigm glass. A sample of questions to consider is the following: 
- What will I learn about the situation (e.g. actors, relationships) if I regard it from this paradigm view?

- What will other participants learn about the situation if they regard it from this paradigm view?

- How does this learning improve my capability to elicit requirements effectively?

- What role do I need to play in the process when I put on this paradigm glass?

The closest we find to this trail of ideas in requirement engineering is Katina et al (2014) share Jackson's view and present a framework for initiating thinking about the problem and the requirement engineering process. The frame includes three phases of understanding the nature of the system observers (e.g. owners, designer, and user) in terms of their different philosophical paradigms, identifying the nature of the system requirements, and the assessment of the nature of the environment. The framework highly abstract and is not supported by methods and tools. Yet, it can still be used as a thinking tool for promoting creativity.

Another useful work is that Minger's multi-methodology method. The multi-methodology method rests on two key ideas (Mingers and Brocklesby, 1997). First, problematic situations exist in the context of three dimensions or worlds: social, personal, material (Habermas, 1997). Second, knowledge about the three dimensions is required and varies along the different phases of the inquiry process. The social world relates to the intersubjective world, which depends on humans but cannot be attributed to any particular person, such as norms, culture, and power. The personal world relates to subjective views shaped by our own personal attributes, such as: perceptions and beliefs. The material world related to the physical objects whose existence and operation is independent from any social or personal aspects, such as mechanics and engineering principles. Outside the systems thinking literature, there are several approaches and tools to boost creativity, such as: lateral thinking (De Bono, 1989).

\subsection{Choice phase}

In the choice phase, the analyst chooses the appropriate methodology, methods, and tools to use through the problem framing process. Although the analyst needs to have an understanding of the project's requirements and methods to be used for problem framing, this does not mean that the whole process has to be sealed at the beginning of the process. Taking a systemic perspective implies the ongoing learning-oriented nature of the inquiry process. As the situation change and new issues emerge, the analyst may like to adopt another epistemological position and/or employ other methods as he/she sees appropriate.

In the choice phase, the analyst is encouraged to make use of methods which helps reveal the strengths and weakness of methodologies with respect to a particular context and task. Critical systems thinking methods, such as critical design of methods and Ulrich's systems heuristics, are useful toolbox for the analyst at this phase. At the heart of critical systems thinking lays the importance to pursue a reflective exploration of judgments and underpinning assumptions in an appropriately purposeful manner rather than ad hoc.

Critical systems thinking methods are designed to help practitioners see through their implicit assumptions, and appreciate the effects of their judgments. On using critical systems thinking, the analyst is asked to frame every choice made through the inquiry process (e.g. which model to use, which stakeholder group to engage) as a boundary judgment which determines what to be included and excluded from the inquiry process. Then, the analyst engages in a reflective practice to think of the implications of this boundary, identify the selectivity biases underpinning judgments, and question whether judgments need to be reviewed. The means to conduct the reflective practice differ among methods. For example, Ulrich (1989) systems heuristic provides a systematic process of 12 questions that get the analyst and other participants involved in the process design think through and communicate about their boundary judgments. Contrasting answers among questions and participants open a window for the analyst to challenge fore-granted assumptions, appreciate limitations, and ideally think of ways to mitigate negative effects (e.g. the risk of marginalizing a particular viewpoint). 


\subsection{Implementation phase}

In the implementation phase, the analyst employs the selected methods in order to define the problem, establish needs, and identify requirements. The framework, following Jackson's critical system practice, deliberately does not offer any specific or detailed description of what/how implementation is carried out. The what (methodology) and how (methods/tools) questions are left to the analyst to answer depending on the context and available methods. In other words, the specific and detailed methodology is seen as an outcome as well as an inquiry process resulting from analyst's practice (including creativity, choices, and implementation). In these terms, theory and practice becomes closely interweaved in the course of a purposeful inquiry with neither taking precedence (Checkland 1985).

Instead of offering a particular methodology description, we prefer to talk in terms of the purposeful orientation of methodologies and methods. Here, we are reminded by distinction made by Churchman's (1968) between purposeful and purposive. Purposeful indicates that purpose can change and develop in the course of the process, while purposive indicates that purpose is fixed and being imposed. Reynolds and Holwell (2010) suggest three generalized purposeful orientations:

- Purposeful orientation 1 is related to making sense, understanding, and simplifying complex relationships between various entities in the situation.

- Purposeful orientation 2 is related to eliciting, surfacing, and engaging (through practice) different perspective related to the situation.

- Purposeful orientation 3 is related to exploring and reconciling issues of boundary setting, power, and potential conflict among different entities and perspectives.

Reynolds and Holwell (2010) argue that different methods have different strengths in supporting these orientations. For example, cognitive mapping is suited to surfacing various perspectives. We used the logic of the three purposeful orientations to analyze a suite of systems thinking methodologies into a catalogue (See Figure 2 for an extract). The catalogue aims to inform the analyst into how to design and implement his/her own purposeful practice.

- Method: cognitive mapping

- Description: Interviews with stakeholders, Construct maps into means-ends representation, Maps analysis and validation

- Possible purposeful actions: Causal analysis of the systemic problem structure, Identify implicit assumptions tacit needs that stakeholders may not be aware of, Compare different views about the problem and solution

- Output: Individual and collective maps

- Strengths: Provides more structured and formal representation than common mind mapping techniques, Supports sophisticated structural analysis features, which provides a robust basis for analysing complexity sources (e.g. feedback loops, delays), Explicit account of uncertainties and ambiguities about concepts and relationships through the use of colours and different types of links.

- Limitations: The mapping and analysis process may be time consuming, the resulting maps are very complex. Some people may find them overwhelming.

- Outcome: Gain visual insight into the: key issues dominant among stakeholders as well as differences, source of conflicts among stakeholders, information gaps, cognitive biases, and flawed assumptions

- Tool-based: For small size maps (<100 nodes), any mapping tool(MS Visio). For large size maps Banxia Decision Explorer tool

- Analyst role (analyst, facilitator, leader): analyst

- Essential skills and knowledge: Interviewing skills, mapping skills

- Potential for combining with other methods (e.g. modelling): The cognitive maps can be used as a conceptual basis for defining scenarios and formulating simulation models (e.g. system dynamics, and agent based model)

\section{Figure 2: An extract from the systems thinking methods catalogue (method: cognitive mapping)}

\section{DISCUSSION AND CONCLUSION}

Here, we discuss some of the points that we think crucial for this paper. First, we do not intend to claim that the presented systems thinking perspective are either superior to those already used in traditional requirement engineering, or silver bullets to framing complex problems. If we do so, then we would have lost our critical (meaning commitment to self-reflection and paradigm critique) systems thinking viewpoint and fell into the fallacy that there is a single method, approach, or a field have a full answer to the complexity question. 
Second, someone may argue correctly that the paper informs understanding about available methods, but does not give a very deep insight for understanding and using each of these methods can be used in practice. In light of the paper's aim, our purpose is to give sufficiently broad insight to raise awareness and trigger interest about these methods. We don't think a single paper can provide a detailed understanding of these methods. What this paper offers is a meaningful and useful point of entry for the systems thinking literature for those interested in further exploration. Third, we acknowledge that the reader's first reaction towards the ideas presented in this paper is perhaps confusion and being overwhelmed by the complexity of presented ideas. Whereas this may be caused by our inability to present the case clearly, we argue there may be deeper explanation for this reaction. This paper aims to promote epistemic learning, as which is described by (Salner, 1986) aim for deliberative break-down and restructuring of mental models that inform worldviews. According to (Prigogine and Stengers, 1994) 'dissipative theory', epistemic learning is a dynamic process, where learners go through a period of confusion and being overwhelmed with complexity before new conceptual knowledge brings in a spontaneous change into more complex mental models, allowing the learner to grasp concepts that we initially unclear. This shift in understanding requires learners to have a genuine openness for learning, exploration, and being intellectually challenged. As we have argued earlier, the aim of this paper is to provide a useful departure point for further exploration. Finally, the presented framework is theory-driven, and has not been applied and tested in a real-life systems engineering applications. Future applications of the framework will help improve the framework based on empirical understanding of 'what works' and 'what does not work' in 'what context'.

\section{REFERENCES}

Ackoff, R. (1979). The Art of Problem Solving: Accompanied by Ackoff's Fables. Wiley.

Baxter, G., \& Sommerville, I. (2011). Socio-technical systems: From design methods to systems engineering. Interacting with Computers, 23(1), 4-17.

Bickerton, M. J. and Siddiqi, J. (1993, January). The classification of requirements engineering methods. In Requirements Engineering, 1993., Proceedings of IEEE International Symposium on (pp. 182-186). IEEE.

Bowers, T. D. (2012). Towards a framework for multiparadigm multimethodologies in systems thinking and practice. Doctoral dissertation, University of Hull.

Checkland, P.B. (1985) From optimizing to learning: a development of systems thinking for the 1990s, Journal of the Operational Research Society, 36: 757-67.

Checkland, P (2000). Soft systems methodology: a thirty year retrospective. John Wiley\& Sons.

Churchman, C. W. (1968). The Design of Inquiring Systems. Basic Books, New York.

Cook, S. C. and Ferris, T. L. (2007). Re-evaluating systems engineering as a framework for tackling systems issues. Systems Research and Behavioral Science, 24(2), 169-181.

De Bono, E. (1989). Six thinking hats. London: Penguin.

Habermas, J., 1979. Communication and the Evolution of Society. Heinemann, London.

Herrmann, N. (1996). The whole brain business book. McGraw Hill Professional.

Hitchins, D. (2015). An introduction to systems thinking. http://www.hitchins.net/systems/systemsthinking/systemsthinking.pdf

Hitchins, D. (2007). Systems engineering: A 21st century methodology. Wiley.

Jackson, M. C. (2006). Creative holism: A critical systems approach to complex problem situations. Systems Research and Behavioral Science, 23(5), 647-657.

Katina, P. F., Keating, C. B., and Ra'ed, M. J. (2014). System requirements engineering in complex situations. Requirements Engineering, 19(1), 45-62.

Kasser, J., \& Mackley, T. (2008, June). Applying systems thinking and aligning it to systems engineering. In INCOSE International Symposium (Vol. 18, No. 1, pp. 1389-1405).

Mingers, J. (2000). Variety is the spice of life: combining soft and hard OR/MS methods. International Transactions in Operational Research, 7(6), 673-691.

Mingers, J. and Brocklesby, J., 1997. Multimethodology: towards a framework for mixing methodologies. Omega 25(5), 489-509.

Prigogine, I. and Stengers, I. (1984) Order Out of Chaos: Man's New Dialogue with Nature. London: Heinemann.

Reynolds, M. and Holwell, S. (2010). Introducing systems approaches. In Systems approaches to managing change: A practical guide (pp. 1-23). Springer London.

Salner, M. (1986). Adult cognitive and epistemological development in systems education. Systems Research. 3: 225-32 